\title{
Fracture zone imaging by using radar tomography \\ - Numerical study by the gradient method -
}

\author{
Yoshinori Sanada, Toshihumi Matsuoka, \\ Keisuke Yamamoto, and Yuzuru Ashida \\ Graduate School of Engineering, Kyoto University, Kyoto, 606-8501, Japan. \\ e-mail : sanada@tansa.kumst.kyoto-u.ac.jp
}

\begin{abstract}
In this paper we show the numerical study of the fracture zone imaging by the radar tomography. The sources are arranged in one bore-hole and the receivers are in another bore-hole. In order to image the conductive distribution between these bore-holes, the gradient method is adopted. It estimates from observed electric field data by using a gradient search for the minimum of error function, and does not require a integral equation or simultaneous equations. The advantage of this inversion scheme is to solve the revised subsurface model quite simply, if we have a forward modeling simulator. The modeling simulator using the Fourie transform based on the FDTD (Finite Difference Time Domain) method is adopted for this simulator since it has the accuracy of the computation results, relatively high speed calculations and small memory space. Numerical studies were carried out and we found that the shape of the fracture zone is recognized clearly after 30 iterations.
\end{abstract}

\section{Introduction}

The investigation of the fracture zone is very important for safe disposal of spent nuclear fuel, civil and environmental engineerng (Olsson,1992). The main object of the inversion procedure to investigate the resistivity distributions by EM methods has becu ardopted to explore the large-scale metal deposit and the regional geological structure. But recently, the high quality method to estimate the distribution of the rock strength and the underground water is demanded in the field of civil and environmental engineering. These inversion methods are classified into roughly two groups, which are the method in the frequency-domain and in the time-domain. Due to the high technology of the instrument to acquire the data, we can obtain the time series lata in the small sampling rate, so the time-domain method is now studied lively. Wang et al. (1994) conducted the imaging of the conductivity and the permeability to the Transient Electromagnetic Method (TEM) 
data and Matsuoka et al. (1999) to GPR data. This method is based on the thought of full wave inversion for seismology data (Tarantora, 1984). The algorithm of this method is a gradient search for minimum of the error function, and need not to use the complex integral equation and or simultaneous equations. The most advantage of this inversion scheme is to solve the revised subsurface model quite simply, if we have a forward modeling simulator.

This inversion method recunires many repetitions of the forward modeling such as, we conduct forward calculations of the electric field for the given estimated model, and the back propagation of the errors between the observed and the calculated field. And every repetition of the calculations, we need to retain the electric data at all grids for every time steps. Therefore the computation time of this inversion method is greatly depend on the number of the grid points. So, if the number of the grid in the forward calculation is decreased, the time of this inversion method is greatly short.

Recently, The modeling simulator using the Fourie transform based on The FDTD method (Yee, 1966) is developed, which is one of the simulation methods of Maxwell's equations (Sanada et al., 1999a, 1999b). This method adopts the staggerd grid, and resolves the partial derivative calculation by the central-difference algorithm in the time, while those in the space by the Fourie method explicitly, we can conduct the simulation of the electromagnetic wave speedy and compactly.

The object of this study is to apply this inversion methodology to the radar tomography and to estimate the subsurface distribution of conductivity values. We assume that the permeability is constant and to estimate the conductivity distributions using observed the electric field which is caused by the electromagnetic wave propagated between two bore-holes.

\section{The EM full wave inversion}

This inversion scheme solves the revised subsurface model quite simply, if we have a forward modeling simulator. The modeling simulator using the Fourie transform based on The FDTD method is adopted for this simulator since it has the accuracy of the computation results, relatively high speed calculations and small memory space.

\subsection{The Simulator}

We consider a two-dimensional problem, which has the z-component electric field and the $\mathrm{x}$ - and $\mathrm{y}$-component magnetic fields in a isotopic media. The Maxwell's equations with current source $\mathbf{J}$ are as follows.

$$
\begin{aligned}
\mu \frac{\partial \mathbf{H}_{x}}{\partial \dot{t}} & =-\frac{\partial \mathbf{E}_{z}}{\partial y} \\
\mu \frac{\partial \mathbf{H}_{y}}{\partial t} & =\frac{\partial \mathbf{E}_{z}}{\partial x}
\end{aligned}
$$




$$
\varepsilon \frac{\partial \mathbf{E}_{z}}{\partial t}=\frac{\partial \mathbf{H}_{y}}{\partial x}-\frac{\partial \mathbf{H}_{x}}{\partial y}-\sigma \mathbf{E}_{z}
$$

where $\mathrm{H}, \mathrm{E}, \mu, \varepsilon$ and $\sigma$ are the magnetic field strength, the electric field strength, the magnetic permeability, the electrical permittivity and the electric conductivity (reciprocal of resistivity), respectively. In this method the first derivatives with respect to the time

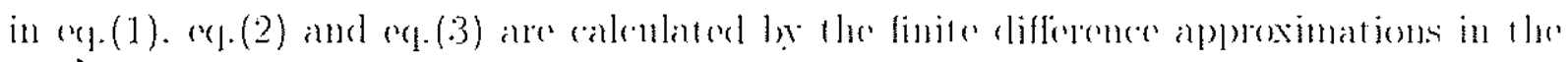
sance way of the FDTD mothod. While those with respere to the space are calculated by the Fourie method in the same way of the Pseudo-spectrum method.

First, a two-dimensional staggered grid of Yee (Yee, 1966) is shown in Fig.1. We define function by equation (4).

$$
\mathbf{F}(i \Delta x, j \Delta y, k \Delta z, n \Delta t)=\mathbf{F}^{n}(i, j, k)
$$

where $\Delta x, \Delta y$ and $\Delta t$ are the grid space and the time increment, respectively. Tho finite difference approximations by central differences are expressed as follows.

$$
\begin{gathered}
\frac{\partial \mathbf{H}_{x}}{\partial t}=\frac{\mathbf{H}_{x}^{n+\frac{1}{2}}\left(i, j+\frac{1}{2}\right)-\mathbf{H}_{x}^{n-\frac{1}{2}}\left(i, j+\frac{1}{2}\right)}{\Delta t} \\
\frac{\partial \mathbf{H}_{y}}{\partial t}=\frac{\mathbf{H}_{y}^{n+\frac{1}{2}}\left(i+\frac{1}{2}, j\right)-\mathbf{H}_{y}^{n-\frac{1}{2}}\left(i+\frac{1}{2}, j\right)}{\Delta t} \\
\frac{\partial \mathbf{E}_{z}}{\partial t}=\frac{\mathbf{E}_{z}^{n+1}(i, j)-\mathbf{E}_{z}^{n}(i, j)}{\Delta t}
\end{gathered}
$$

The last term $\mathrm{E}_{z}$ in eq. 3 is clefined as follows.

$$
\mathrm{E}_{z}=\frac{\mathrm{E}_{y}^{n+1}(i . j)+\mathrm{E}_{\eta}^{n}(i, j)}{\underline{2}}
$$

In the space derivative is calculated by Witte's first derivative operator (Witte, 1990).

$$
\begin{aligned}
& \frac{\partial \mathrm{E}_{z}}{\partial y}=\frac{1}{N} \sum_{n=0}^{N-1} j n \Delta k_{y} \exp \left(j n \Delta k_{y} \Delta y / 2\right) \tilde{\mathrm{E}}_{z} \operatorname{(xp}(j 2 \pi m n / N)
\end{aligned}
$$

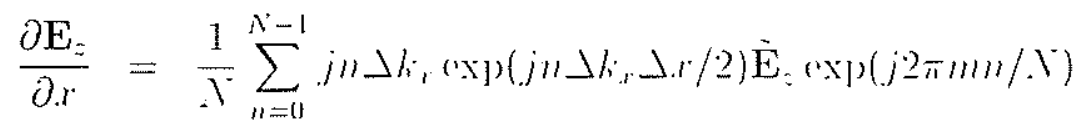

$$
\begin{aligned}
& \frac{\partial \mathbf{H}_{y}}{\partial x}=\frac{1}{N} \sum_{n=0}^{N-1} j n \Delta k_{r r}\left(\operatorname{xp}\left(-j n \Delta k_{x} \Delta x / 2\right) \hat{\mathbf{H}}_{y}(\operatorname{xp})(j 2 \pi m n / V)\right.
\end{aligned}
$$

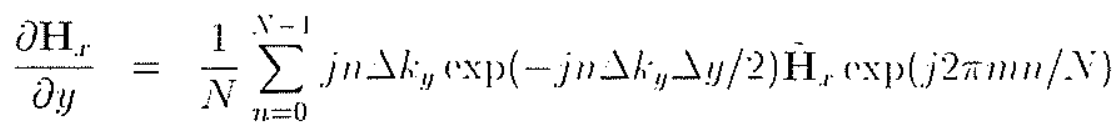

We get these processes in shape. 
1. Take FFT of $\mathrm{E}_{z}$ with respect to $\mathrm{x}$ and $\mathrm{y}$.

2. Multiply $\left.\tilde{\mathrm{E}}_{z}\right|_{x},\left.\quad \tilde{\mathrm{E}}_{z}\right|_{y}$ by $j n \Delta k_{x} \exp \left(j n \Delta k_{x} \Delta x / 2\right)$ and $j n \Delta k_{y} \exp \left(j n \Delta k_{y} \Delta y / 2\right)$, respectively.

3. Take IFFT to obtain the space parcial derivative of Ez.

4. Advance time step by the finite difference in time parcial derivative.

5. Take FFT of $\mathrm{H}_{3}$ with respert to $x$. $\mathrm{H}_{\text {, with respect to }}$.

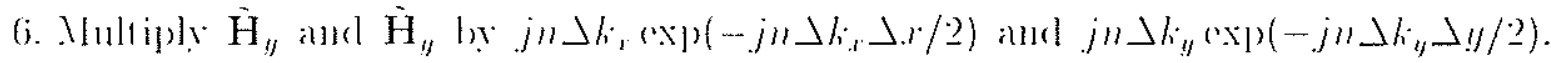
respectively.

7. Take IFFT to obtain the space parcial derivative of $\mathrm{Hx}$ and $\mathrm{Hy}$.

8. Advance time step by the finite difference in time parcial derivative.

9. Repeat the 1 . to 8 . operations till the end of time steps.

\subsection{The inversion algorithm}

Only the last solutions of the inversion are shown here. The detail processes are referenced Matsuoka et al. (1999).

$$
\begin{aligned}
\sigma^{(n+1)} & =\sigma^{(n)}-\alpha_{n} \gamma^{(n)} \\
\gamma\left(\mathbf{r}^{\prime}\right) & =\int_{0}^{T} d t^{\prime} \mathbf{E}\left(\mathbf{r}^{\prime}, t^{\prime}\right) \cdot \mathbf{E}_{b}\left(\mathbf{r}^{\prime}, t^{\prime} \mid \delta \mathbf{E}^{0}\right)
\end{aligned}
$$

Here, $n$ is the mumber of the iteration, and $\alpha$ is the step length. At the first step of the itcration, we determine the first distribution of conductivity values (first model) as we like. Here, the $\mathrm{E}_{b}\left(\mathbf{r}^{\prime}, t^{\prime} \mid \delta \mathrm{E}^{0}\right)$ is as follows.

$$
\begin{gathered}
\mathrm{E}_{b}\left(\mathbf{r}^{\prime}, t^{\prime} \mid \delta \mathbf{E}^{0}\right)=\sum_{i} \int_{T}^{t^{\prime}} d t \mathscr{G}_{11}^{+}\left(\mathbf{r}^{\prime}, t^{\prime} \mid \mathbf{r}_{i}, t\right) \cdot \delta \mathbf{E}^{0}\left(\mathbf{r}_{i}, t\right) \\
\delta \mathrm{E}^{0}\left(\mathbf{r}_{i}, t\right)=\mathrm{E}^{0}\left(\mathbf{r}_{i}, t\right)-\mathrm{E}\left(\mathbf{r}_{i}, t\right)
\end{gathered}
$$

Here, according to eq. $(15), \delta \mathbf{E}^{0}\left(\mathbf{r}_{i}, t\right)$ is the errors between the observed data in a site and at the estimated model, and $\mathscr{G}_{11}^{+}$is the adjoint dyadic Green function. So, the physical meaning of $\mathbf{E}_{b}\left(\mathbf{r}^{\prime}, t^{\prime} \mid \delta \mathbf{E}^{0}\right)$ is the electric data at every receiver point caused by back propagation in reverse time of the errors between the observed data in a site and simulation lata for the estimated model. And, $\mathrm{E}\left(\mathbf{r}^{\prime}, t^{\prime}\right)$ is the estimated electric data occurred by the

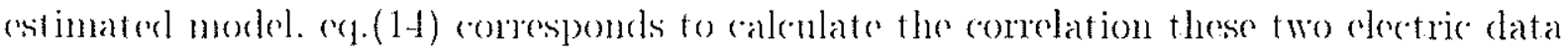
at all the grid points. Zlidanov (1993) and Wang at al. (1994) showed that the scheme in the reverse time for the adjoint equation is same as in the forward time for the diffusion equation, and it is stable. So, we can conduct the inversion by using the stable forward simulator. At last. wo define the error finctional as the following equation because of the judge of the modification of the motel.

$$
E r r=\frac{\sum_{\text {source receiner }}\left(\mathrm{e}^{0}-\mathrm{e}\right)^{2}}{\sum_{\text {source receiver }} \sum_{\text {ret }}\left(\mathrm{e}^{0}\right)^{2}}
$$


And, we repeat the modification until the error functional is regarded as the convergence.

We get these ideas in shape.

1. At the assumed model, by some sources, we evaluate the electric field $\mathbf{E}\left(\mathbf{r}^{\prime}, t^{\prime}\right)$ at all the grid points in every time steps and the observed electric field data $\mathbf{E}\left(\mathbf{r}_{i}, t\right)$ at all the receiver points.

2. We calculate the errors $\delta \mathbf{E}^{0}\left(\mathbf{r}_{i}, t\right)$ between the observed electric data in a site $\mathbf{E}^{0}\left(\mathbf{r}_{i} . t\right)$ and for the estimated model at the receiver point, propagate these errors back into the model in reverse time, and calculate the electric data $\mathrm{E}_{b}\left(\mathbf{r}^{\prime}, t^{\prime} \mid \delta \mathrm{e}^{0}\right)$ at all the grid points in every time steps.

3. At all the grid points, we calculate the cross correlation $\mathbf{E}\left(\mathbf{r}^{\prime}, t^{\prime}\right)$ with $\mathbf{E}_{b}\left(\mathbf{r}^{\prime}, t^{\prime} \mid \delta \mathbf{E}^{0}\right)$, calculate the amount of the modification, and modify the conductivity of the model.

4. We repeat the 1 . to 3 . operations until the error functional converges on some value.

\section{Numerical examples}

In this time, we conduct the simulation study. We adopt a model which has $0.001 \mathrm{~S} / \mathrm{m}$ background conductivity for $20 \mathrm{~m}$ around, and $0.01 \mathrm{~S} / \mathrm{m}$ conductivity anomaly of the fracture zone. Fig. 2 shows this model which we studies. We arrange the 11 sources in the bore-hole at $20 \mathrm{~m}$ intervals between (distance, depth) $=(0 \mathrm{~m}, 0 \mathrm{~m})$ and $(0 \mathrm{~m}, 20 \mathrm{~m})$, the 11 receivers in the bore-hole at $2 \mathrm{~m}$ intervals between (distance, depth) $=(0 \mathrm{~m}, 20 \mathrm{~m})$ and $(20 \mathrm{~m}$, $20 \mathrm{~m}$ ). In this study, we give the $0.001 \mathrm{~S} / \mathrm{m}$ homogeneous medium as the first model. Fig. 3 (a) shows the result after 10 time iteration, Fig. 3(b) shows the result after 30 times iterations, and Fig. 4 shows the error functional. We can clearly identify the fracture zone. However, the estimated value of the conductivity is smaller than the true value. Fig. 4 shows that the error functional takes the turn for the convergence at the 30 iterations.

\section{Conclusions}

In this paper, we show the numerical study of the fracture zone imaging for a bore-hole to bore-hole EM tomography. We arrange the sources in one bore-hole and the receivers in another bore-hole. And, we can estimate the subsurface distributions of the conductivity from the observed electric field data by the inversion method using gradient method. From the simulation studies, we concluded that

1: the shape of the fracture zone is recognized clearly after 30 iterations.

2: The estimated value of the conductivity is smaller than the true value.

We can showed only the simulation studies, however, the further investigations are necessary such as;

1: change the position of the sources and receivers and consider the more different arrangements.

2: developing a method for the reduction of the calculated time since it still required 
long computation time.

3: apply to the real data in a site, and compare with the other inversion method.

\section{References}

[1] Matsuoka, T.. Siluada, Y.. Yamamoto, K. and Ashida, Y. (1999) : Inversion of EM tomography by gradient method, Proc. of the Soc. of professional well log analysts the fifth well logging symposium of Japan, Paper $\mathrm{J}$.

[2] Olsson, O, Falk, L, Forslund, O, Lundmark, L, and Sandberg, E (1992) : Borehole radar applied to the characterization of hydraulically conductive fracture zones in crystalline rock, Geophysical prospecting, 40, 109-142.

[3] Sanada, Y., Matsuoka, T. and Ashida, Y. (1999a) : EM wave modeling by finite difference time domain method using Pseudo spectral method, Proc. of the 100th SEGJ Conference, 232-235.

[4] Sanada, Y., Matsuoka, T. and Ashida, Y. (1999b) : EM wave modeling by finite difference time domain method using Pseudo spectral method (2), Proc. of the 101st SEGJ Conference, 197-200.

[5] Tarantola, A., (1984) : Inversion of seismic reflection data in the acoustic approximation, Geophysics, 49, no. 8, 1259-1266.

[6] Witte, D. C., and Richards, P. G. (1990) : The pseudospectral method for simulating wave propagation, in Lee, D., Cakmak, A. and Vichnevetsky, R. ed., Computational Acoustics vol. 3, Elsevier Science Publisher, North-Holland, 1-18.

[7] Wang, T., Oristaglio, M., Tripp, A., and Hohmann, G. (1994) : Inversion of diffusive transient electromagnetic data by a conjugate-gradient method: Radio Science, 29, 1143-1156.

[8] Yee, K. S., (1966) : Numerical Solution of Initial Boundary Value Problems Involving Maxwell's Equations in Isotropic Media, IEEE Trans. Antennas and Propagat., AP14, 302-307.

[9] Zhdanov, M. S. and Booker, J. R., (1993) : Underground imaging by electromagnetic migration, in Expanded Abstracts, 63rd Annual International Meeting, Society of Exploration Geophysics: Tulsa, Okla., 355-357. 


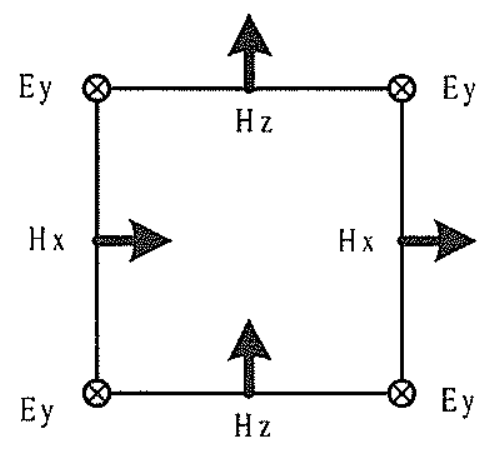

Fig. 1 Staggerd grid (Yee, 1966)
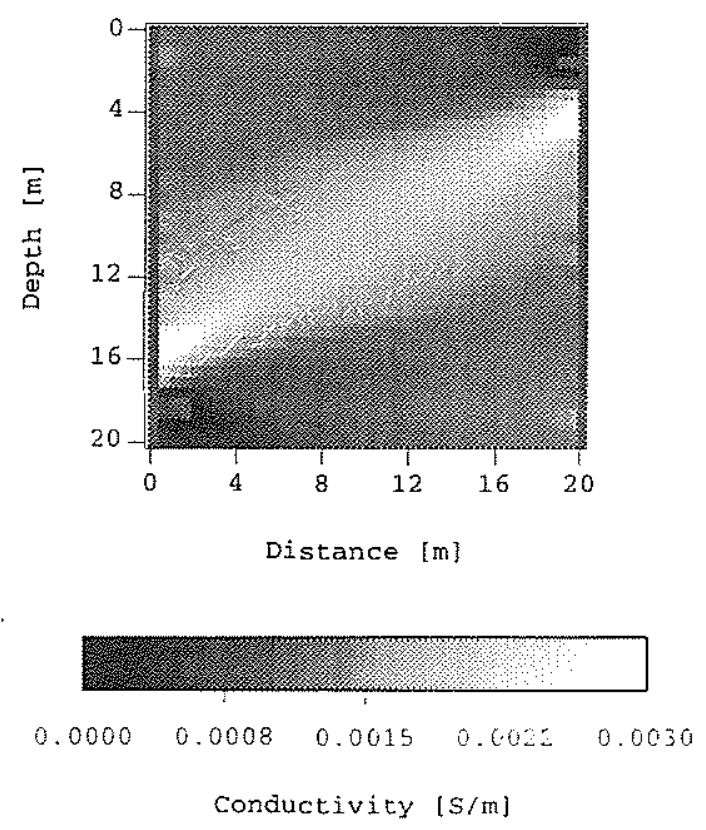

(a)

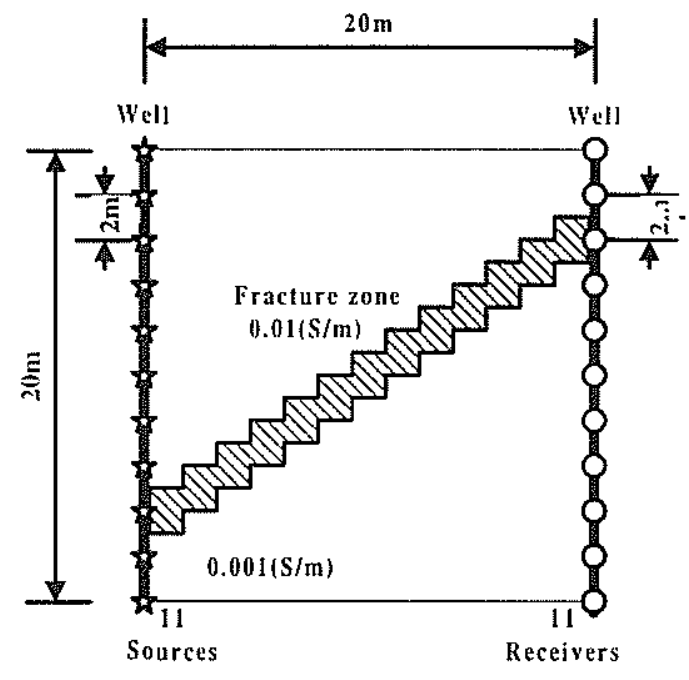

Fig. 2 Fracture zone model.
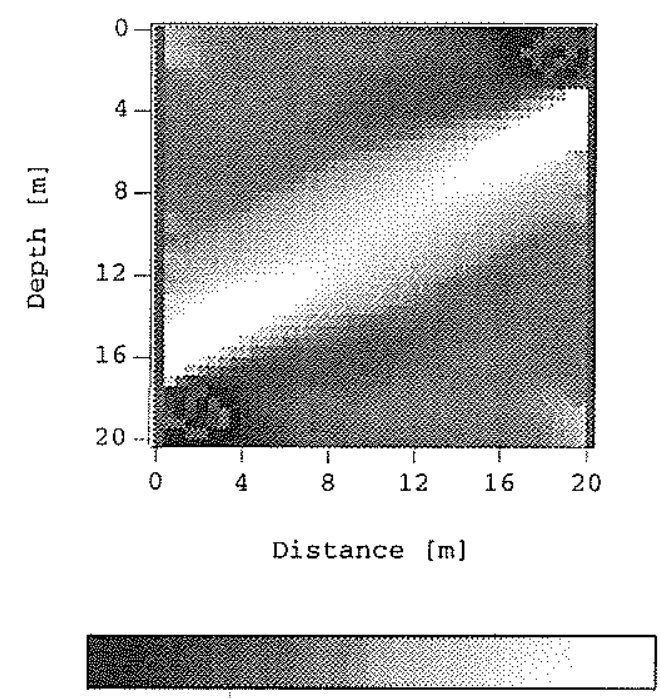

$0.0000 \quad 0.0008 \quad 0.0015 \quad 0.002=$ Conductivity $[\mathrm{s} / \mathrm{m}]$

(b)

Fig.3 Inversion results. (a) 10 iteraion. (b) 30 iteration.

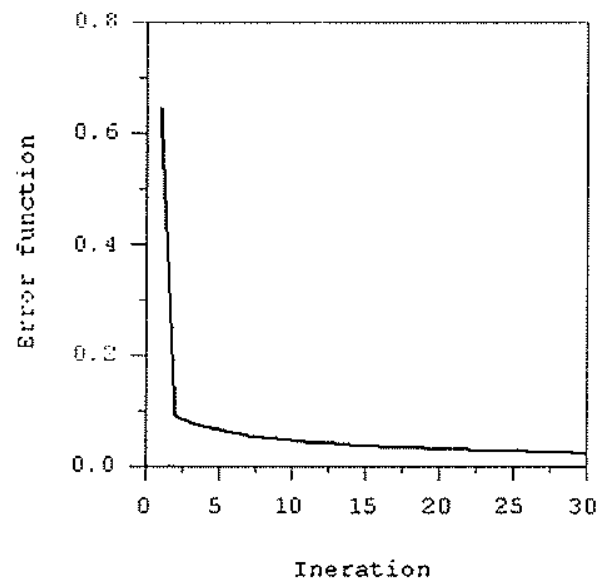

Fig. 4 Error function. 